\title{
REDUCTION THEOREMS FOR A CLASS OF SEMILINEAR EQUATIONS AT RESONANCE
}

\author{
PETER W. BATES
}

\begin{abstract}
In solving equations of the form $L u-N u=p$ in a Hilbert space, where $L$ is linear and $N$ is nonlinear, the alternative method can sometimes be used to reduce the problem to one in a subspace. In this note previous reduction results are extended and at the same time the proofs are simplified. The approach is to use simple fixed point theorems in place of the traditional variational methods which are often quite delicate.
\end{abstract}

1. Introduction. This note is to extend previous results by Castro [6], Bates and Castro [3], Amann [1], Bates [2] and Mawhin [7, 8], while giving simpler proofs of the results in $[1,2,3]$. An application of the abstract results shows that under certain assumptions on $g$, the equation

$$
y^{\prime \prime}+g(t+y)=c=\text { constant }
$$

has no $2 \pi$-periodic solutions if $c \neq 0$ and if $c=0$ has a continuum of such solutions. This example was chosen because it seemed interesting; it is not a general representative of the class of equations treated here. In fact the abstract results below may be applied to semilinear elliptic and hyperbolic PDE's.

To proceed, let $H$ be a (real or complex) Hilbert space with inner product $(\cdot, \cdot)$ and norm $\|\cdot\|$, let $L$ be a linear operator in $H, N$ be a nonlinear operator on $H$ and let $p \in H$. Consider the question of solvability of

$$
L u-N u=p .
$$

In [7] Mawhin has

THEOREM A. Suppose $L$ is selfadjoint with spectrum $\sigma$ and $N$ has a selfadjoint Gâteaux derivative $N^{\prime}(u)$ which satisfies

(1.2) There exist numbers $a \leqslant b$ with $[a, b] \cap \sigma=\varnothing$ and $a I \leqslant N^{\prime}(u) \leqslant b I$ for each $u \in H$.

Then (1.1) is uniquely solvable with the solution depending (Lipschitz) continuously on $p$.

Received by the editors February 11, 1981; presented to the Society at the 87th annual meeting, January 9, 1981.

1980 Mathematics Subject Classification. Primary 47H15, 47H17, 34C25; Secondary 47H09, 34B15, 35B, 35J, 35L.

Key words and phrases. Hilbert space, spectrum, nonexpansive map, periodic solutions of nonlinear ordinary differential equations. 
The main theorem of this paper will contain as a special case

THEOREM 1. Suppose $L$ and $N$ are as above except (1.2) is replaced by:

(1.2)' For each $u \in H$ there exist numbers $a(u)<b(u)$ with $[a(u), b(u)] \cap \sigma=\varnothing$ and such that $a(u) I<N^{\prime}(u) \leqslant b(u) I$.

(1.2)" $-\infty<a \equiv \inf \{a(u): u \in H\}<\sup \{b(u): u \in H\} \equiv b<\infty$ and for some constants $C>0, d<(b-a) / 2$,

$$
\|N(u)-(a+b) u / 2\|<d\|u\|+C .
$$

Then (1.1) has a unique solution.

REMARK. The value of Theorem 1 over Theorem $A$ is that $[a, b] \cap \sigma \neq \varnothing$ is allowed; that is, the union of the numerical ranges of the operators $N^{\prime}(u), u \in H$, may have points of $\sigma$ in its closure.

Whereas the above theorems are existence results we are mainly concerned here with reduction theorems associated with applying the alternative method to (1.1). To be specific, suppose $H$ has the orthogonal decomposition $H=H_{1} \oplus H_{2}$, with $L$ leaving $H_{i}$ invariant. Let $P_{i}$ be the orthogonal projection onto $H_{i}$; then (1.1) is equivalent to the system

$$
\begin{aligned}
& L u_{1}-P_{1} N\left(u_{1}+u_{2}\right)=p_{1}, \\
& L u_{2}-P_{2} N\left(u_{1}+u_{2}\right)=p_{2},
\end{aligned}
$$

where for $z \in H, z_{i}=P_{i} z \in H_{i}$. Under certain hypotheses on $L$ and $N$ it may be possible to solve (1.3b) once $u_{1}$ is fixed, in which case solvability of (1.1) is reduced to solvability of (1.3a) with $u_{2}=u_{2}\left(u_{1}\right)$ being the solution of (1.3b). Thus, we are concerned with imposing conditions on $L$ and $N$ so that (1.3b) has, for fixed $u_{1}$, a unique solution $u_{2}\left(u_{1}\right)$ which depends continuously on $u_{1}$. Theorems $\mathrm{B}$ and $\mathrm{C}$ below are along these lines and are extended in Theorem 2 . Suppose

(1.4) $L$ is selfadjoint with spectrum $\sigma \subset(-\infty, a-\varepsilon] \cup[a, b] \cup[b+\varepsilon, \infty)$, where $a \leqslant b$ and $\varepsilon>0$;

(1.5) $N$ is a continuous gradient operator such that $a\|u-v\|^{2}<(N u-N v$, $u-v)<b\|u-v\|^{2}$ for $u, v \in H$.

Now suppose $\left\{E_{\lambda}\right\}$ is the resolution of the identity associated with $L$ and let $P_{1}=\int_{a}^{b} d E_{\lambda}, P_{2}=I-P_{1}$ and $H_{i}=P_{i} H, i=1,2$.

TheOREM B (AMANN [1]). Suppose (1.4) and (1.5) hold. Then for each $u_{1} \in H_{1}$, (1.3b) has a unique solution $u_{2}\left(u_{1}\right) \in H_{2}$. Furthermore, $u_{2}$ depends continuously upon $u_{1}$.

REMARK. In [1] Amann gave a variational proof of this result and in [8] Mawhin has since given a much simpler proof. It was Mawhin's proof which inspired this note.

A different theorem founded in [6] and improved in [3] and [2] involves weakening the hypotheses on $N$ and strengthening those on $L$. Suppose

(1.6) The restriction of $L$ to $H_{2}$ has compact resolvent,

(1.7) $N$ is a continuous gradient operator such that, for $u \neq v,(a-\varepsilon)\|u-v\|^{2}$ $<(N u-N v, u-v)<(b+\varepsilon)\|u-v\|^{2}$, and 
(1.8) $\lim \sup _{\|u\| \rightarrow \infty}\|N u-(a+b) u / 2\| /\|u\|<(b-a) / 2+\varepsilon$.

TheOrem C. Suppose L satisfies (1.4) and (1.6) and that $N$ satisfies (1.7) and (1.8). Then the conclusion of Theorem B holds.

Remark. Theorem $\mathrm{C}$ may be used to show that $y^{\prime \prime}+\sin y=p(t)$ has $2 \pi$-periodic solutions for certain functions $p(t)$ where Theorem B fails to be of use.

Now suppose (perhaps after shifting $L$ and $N$ so that $a=-b$ in (1.4)):

(1.9) $H$ has the orthogonal decomposition $H_{1} \oplus H_{2}$ with $H_{i}$ invariant under the closed operator $L$, and such that $\left\|\left(L_{H_{2}}\right)^{-1}\right\|<1 / l$ for some $l>0$;

(1.10) For $u \neq v,\|N u-N v\|<l\|u-v\|$.

(1.11) For some constants $\bar{l}<l$ and $C>0,\|N u\|<\bar{l}\|u\|+C$.

Let $P_{i}$ be the orthogonal projection onto $H_{i}$.

TheORem 2. Suppose that $L$ and $N$ satisfy (1.9)-(1.11). Then for each $u_{1} \in H_{1}$ there exists a unique $u_{2}\left(u_{1}\right) \in H_{2}$ satisfying (1.3b).

Lacking in this theorem is the continuity of $u_{2}(\cdot)$; however, suppose

(1.12) $H_{2}$ has the orthogonal decomposition $H_{3} \oplus H_{4}$ with $H_{i}$ invariant under $L$ $(i=3,4), H_{3}$ finite dimensional and with $\left\|\left(\left.L\right|_{H_{4}}\right)^{-1}\right\|<1 / l$.

We have

THEOREM 3. If (1.9)-(1.12) hold, then the function $u_{2}(\cdot)$ given by Theorem 2 is continuous.

Remarks. 1. Although it was not stated, Theorems $\mathrm{B}$ and $\mathrm{C}$ required $H$ to be real; Theorems 2 and 3 do not.

2. If $L$ is self-adjoint (or normal) satisfying (1.4) (respectively, $\sigma \subset \bar{D}((a+b) / 2$, $(b-a) / 2) \cup D^{c}((a+b) / 2,(b-a) / 2+\varepsilon)$, where $D(z, r)$ is the open disc in $\mathbf{C}$ centered at $z$ of radius $r)$ then $L-(a+b) I / 2$ satisfies (1.9) with $l=(b-a) / 2+$ $\varepsilon$ and $H_{i}$ chosen in the obvious way.

3. Condition (1.7) implies that $N-(a+b) I / 2$ satisfies (1.10) (see [4] or [8]) and (1.11) follows from (1.8).

4. There is no compactness assumption on the resolvent of $L$ and $N$ need not be a gradient operator.

2. Proofs. Theorem 2 has a particularly simple proof using the following result of F. Browder [5]:

THEOREM D. Let $B=\{u:\|u\|<R\}$ and $S=\partial B$. Let $T: B \rightarrow H$ satisfy $\|T u-T v\|<\|u-v\|$ for all $u, v \in B$ and $T(S) \subset B$. Then $T$ has a fixed point in $B$.

Proof of Theorem 2. For fixed $u_{1} \in H_{1}$, rewrite (1.3b) as

$$
u_{2}=L^{-1}\left(P_{2} N\left(u_{1}+u_{2}\right)+p_{2}\right) \equiv T u_{2} \text {. }
$$

Note that

$$
\left\|T u_{2}-T \bar{u}_{2}\right\| \leqslant\left\|\left(\left.L\right|_{H_{2}}\right)^{-1}\right\| \cdot\left\|N\left(u_{1}+u_{2}\right)-N\left(u_{1}+\bar{u}_{2}\right)\right\|<\left\|u_{2}-\bar{u}_{2}\right\|
$$

for $u_{2} \neq \bar{u}_{2}$, by (1.9) and (1.10). Thus, $T$ is nonexpansive and any fixed point is 
unique. Finally, (1.11) implies

$$
\left\|T u_{2}\right\| /\left\|u_{2}\right\| \leqslant \bar{l} / l+\left(\bar{l}\left\|u_{1}\right\|+C+\left\|p_{2}\right\|\right) /\left(l\left\|u_{2}\right\|\right) .
$$

Since $u_{1}$ is fixed, for $\left\|u_{2}\right\|=R$ sufficiently large, $\left\|T u_{2}\right\|<\left\|u_{2}\right\|$. Theorem D completes the proof.

Proof of Theorem 1. Condition (1.2)' implies that for each $u \in H$ there are points $c(u), d(u) \in \sigma \cup\{a, b\}$ such that $(c(u), d(u)) \cap \sigma=\varnothing$ and $c(u)<a(u) \leqslant$ $b(u)<d(u)$. Actually, $c$ and $d$ do not depend upon $u$. This follows from an extension of the "Intermediate Value Theorem for Derivatives" as outlined below. Suppose $c\left(u_{1}\right) \leqslant\left(N^{\prime}\left(u_{1}\right) v, v\right) \leqslant d\left(u_{1}\right)$ and $c\left(u_{2}\right) \leqslant\left(N^{\prime}\left(u_{2}\right) v, v\right) \leqslant d\left(u_{2}\right)$ for all $v \in$ $H$ and suppose $d\left(u_{1}\right) \leqslant c\left(u_{2}\right)$. Then $\left[d\left(u_{1}\right), c\left(u_{2}\right)\right]$ is contained in the set $\left\{\left(N^{\prime}(u) v, v\right): u \in H\right\}$, where $v=u_{2}-u_{1}$. To see this, consider the continuous functions $f, g:(0,1] \rightarrow \mathbf{R}$ defined by

$$
\begin{aligned}
& f(t)=\left(N\left(u_{2}+t\left(u_{1}-u_{2}\right)\right)-N\left(u_{2}\right), u_{1}-u_{2}\right) / t, \\
& g(t)=\left(N\left(u_{1}+t\left(u_{2}-u_{1}\right)\right)-N\left(u_{1}\right), u_{2}-u_{1}\right) / t .
\end{aligned}
$$

By the Mean Value Theorem (see [9]) we may write

$$
f(t)=\left(N^{\prime}(z(t))\left(u_{1}-u_{2}\right), u_{1}-u_{2}\right), \quad g(t)=\left(N^{\prime}(y(t))\left(u_{2}-u_{1}\right), u_{2}-u_{1}\right)
$$

for some points $z(t), y(t)$ lying on the line segment joining $u_{1}$ and $u_{2}$. Now, $f$ and $g$ are continuous at 0 , and defined by $f(0)=\left(N^{\prime}\left(u_{2}\right)\left(u_{1}-u_{2}\right), u_{1}-u_{2}\right), g(0)=$ $\left(N^{\prime}\left(u_{1}\right)\left(u_{2}-u_{1}\right), u_{2}-u_{1}\right)$. Note also that $f(1)=g(1)$, so that $f([0,1]) \cup g([0,1])$ is connected, and by the representation $(2.2),[g(0), f(0)]$ is contained in

$$
\left\{\left(N^{\prime}(u)\left(u_{2}-u_{1}\right), u_{2}-u_{1}\right): u \text { lies on the line segment joining } u_{1} \text { and } u_{2}\right\} \text {. }
$$

For the sake of simplicity suppose $a, b \in \sigma$. In Theorem 2 take $l=(b-a) / 2$, $\hat{L} \equiv L-(b+a) I / 2$ in place of $L, \hat{N} \equiv N-(b+a) I / 2$ in place of $N, H_{1}=\{0\}$, $H_{2}=H, \bar{l}=d$. It is easy to see that (1.9) and (1.11) hold. By the Mean Value Theorem $\|\hat{N} u-\hat{N} v\| \leqslant\left\|\hat{N}^{\prime}(z)\right\| \cdot\|u-v\|$ for some $z$ on the line segment joining $u$ and $v$. Now $\hat{N}^{\prime}(z)$ is selfadjoint and $a-(a+b) / 2<a(z)-(a+b) / 2<$ $\left(\hat{N}^{\prime}(z) v, v\right) \leqslant b(z)-(a+b) / 2<b-(a+b) / 2$, so $\left\|\hat{N}^{\prime}(z)\right\|<(b-a) / 2$ and (1.10) holds. This completes the proof.

Proof of TheOREM 3. Write (1.3b) as

$$
\begin{aligned}
& L u_{3}-P_{3} N\left(u_{1}+u_{3}+u_{4}\right)=p_{3}, \\
& L u_{4}-P_{4} N\left(u_{1}+u_{3}+u_{4}\right)=p_{4},
\end{aligned}
$$

where $u_{2}=u_{3}+u_{4}, p_{2}=p_{3}+p_{4} \in H_{3} \oplus H_{4}$, and $P_{i}$ is the orthogonal projection onto $H_{i}, i=3,4$. Fix $u_{1}+u_{3} \in H_{1} \oplus H_{3}$ and consider (2.4) rewritten as $u_{4}=$ $\left(\left.L\right|_{H_{4}}\right)^{-1}\left(P_{4} N\left(u_{1}+u_{3}+u_{4}\right)+p_{4}\right) \equiv K u_{4}$. From conditions (1.10) and (1.12) it is easy to see that $K$ is a strict contraction, and has, by the Contraction Mapping Theorem, a unique fixed point $u_{4}\left(u_{1}+u_{3}\right)$. Furthermore, $u_{4}: H_{1} \oplus H_{3} \rightarrow H_{4}$ is Lipschitz continuous. Now, from Theorem 2, we know that (2.3), (2.4) has a unique solution for fixed $u_{1}$. Write this as $u_{2}\left(u_{1}\right)=u_{3}\left(u_{1}\right)+\bar{u}_{4}\left(u_{1}\right)$. The uniqueness implies that $\bar{u}_{4}\left(u_{1}\right)=u_{4}\left(u_{1}+u_{3}\left(u_{1}\right)\right)$. Therefore, in order to prove that $u_{2}: H_{1} \rightarrow H_{2}$ is continuous, it suffices to show that $u_{3}: H_{1} \rightarrow H_{3}$ is continuous. From (2.1) it follows that $u_{1} \rightarrow u_{2}\left(u_{1}\right)$ takes bounded sets into bounded sets and so the same is 
true of the mapping $u_{3}(\cdot)$. Suppose that $\left\{u_{1}^{n}\right\} \subset H_{1}$ converges to $u_{1}$. Then since $\operatorname{dim} H_{3}<\infty$ we may assume that a subsequence has been taken so that $\left\{u_{3}\left(u_{1}^{n}\right)\right\}$ converges to a point $u_{3} \in H_{3}$. Since $N$ and $u_{4}(\cdot)$ are continuous and $L$ is closed, it follows that $u_{3}$ and $u_{4}\left(u_{1}+u_{3}\right)$ satisfy (2.3) and (2.4). The uniqueness of the solution implies $u_{3}=u_{3}\left(u_{1}\right)$. It follows that $u_{3}$, and hence $u_{2}$, is continuous.

3. An example. Consider the problem

$$
\begin{gathered}
y^{\prime \prime}+g(y+t)=c=\text { constant, } \\
y(0)-y(2 \pi)=y^{\prime}(0)-y^{\prime}(2 \pi)=0,
\end{gathered}
$$

where $g$ is a differentiable $2 \pi$-periodic function satisfying

$$
\begin{gathered}
\left|g^{\prime}(x)\right| \leqslant 1 \text { for all } x \in \mathbf{R}, \\
\left\{x:\left|g^{\prime}(x)\right|=1\right\} \text { has measure zero, } \\
\int_{0}^{2 \pi} g(s) d s=0 .
\end{gathered}
$$

We will show that for $c \neq 0$ this problem has no solution while for $c=0$ there is a continuum of solutions. Let $H=L_{2}(0,2 \pi), H_{1}=\mathbf{R}, P_{1}$ the projection defined by $P_{1} f=\frac{1}{2 \pi} \int_{0}^{2 \pi} f(x) d x, P_{2}=I-P_{1}, H_{2}=P_{2} H$. Let $L$ be the closure of the operator $L_{0}$ defined by dom $L_{0}=\left\{y \in C^{2}[0,2 \pi]: y(0)-y(2 \pi)=y^{\prime}(0)-y^{\prime}(2 \pi)=0\right\}, L_{0} y$ $=-y^{\prime \prime}$. Then $L$ is selfadjoint with spectrum $\sigma=\left\{k^{2}: k=0,1, \ldots\right\}$ and $\operatorname{Ker} L$ $=H_{1}$. Let $N$ be given by $N(y)=g(y+t)$. Then $N$ is continuous on $H$ and, with $l=1$, satisfies (1.10), for if $y \neq z \in H$,

$$
\begin{aligned}
\|N y-N z\|^{2} & =\int_{0}^{2 \pi}(g(y(t)+t)-g(z(t)+t))^{2} d t \\
& =\int_{0}^{2 \pi}\left[\int_{0}^{1} g^{\prime}(z(t)+t+s(y(t)-z(t))) d s\right]^{2}(y(t)-z(t))^{2} d t .
\end{aligned}
$$

On a set of positive measure $y(t) \neq z(t)$ and hence, for such values of $t$, (3.4) implies $\left|g^{\prime}(z(t)+t+s(y(t)-z(t)))\right|<1$ a.e. for $s \in[0,1]$. This gives (1.10). Using (3.3) and (3.5) it can be shown (integrate by parts) that $|g(x)|<\frac{1}{2}|x|+C$ for $C$ sufficiently large; hence, (1.11) is valid. Clearly (1.9) holds and also (1.12) with $H_{3}=\operatorname{span}\{\sin t, \cos t\}$ and $H_{4}=\overline{\operatorname{span}}\{\sin k t, \cos k t: k=2,3, \ldots\}$. By Theorems 2 and 3, there exists a continuous function $u_{2}: \mathbf{R} \rightarrow H_{2}$ so that (3.1), (3.2) has a solution if and only if there exists a constant $x$ such that

$$
\bar{g}(x) \equiv \frac{1}{2 \pi} \int_{0}^{2 \pi} g\left(t+x+u_{2}(x)(t)\right) d t=c .
$$

We now show that $\bar{g} \equiv 0$, i.e., if $c \neq 0$, (3.1), (3.2) has no solution. This implies that if $c=0, x+u_{2}(x)(t)$ solves (3.1), (3.2) for each $x \in \mathbf{R}$. In general, $u_{2}(x) \neq$ $u_{2}(z)$ when $x \neq z$ so the continuum of solutions is not merely a translate of $\mathbf{R}$ in $H=\mathbf{R}+H_{2}$. Suppose $y$ solves (3.1), (3.2). Multiplying (3.1) by $1+y^{\prime}(t)$ and integrating over $[0, t]$ gives

$$
y^{\prime}(t)+y^{\prime 2}(t) / 2+G(t+y(t))-c y(t)+D=c t .
$$

where $D$ is a constant and $G^{\prime}=g$. Now divide by $t$ and let $t$ tend to infinity. Since

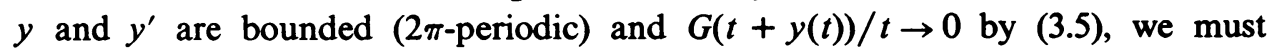


conclude that $c=0$. Actually, this shows that if $c \neq 0$ there are no solutions $y$ of (3.1) for which $y$ and $y^{\prime}$ are bounded.

I would like to thank Professor F. Odeh for pointing out that (3.1), (3.2) has no solutions in the case $c \neq 0$ with $g(x) \equiv \sin x$.

\section{REFERENCES}

1. H. Amann, Saddle points and multiple solutions of differential equations, Math. Z. 169 (1979), 127-166.

2. P. W. Bates, A variational approach to solving semilinear equations at resonance, Proc. Internat. Conf. Nonlinear Phenomena in Math. Sci. (to appear).

3. P. W. Bates and A. Castro, Necessary and sufficient conditions for existence of solutions to equations with noninvertible linear part, preprint, 1979.

4. H. Brezis and L. Nirenberg, Characterizations of the ranges of some nonlinear operators and applications to boundary value problems, Ann. Scuola Norm. Sup. Pisa Cl. Sci. (4) 5 (1978), 225-326.

5. F. Browder, Existence of periodic solutions for nonlinear equations of evolution, Proc. Nat. Acad. Sci. U.S.A. 53 (1965), 1100-1103.

6. A.Castro, Periodic solutions of the forced pendulum equation, Differential Equations (S. Ahmad, M. Keener, A. Lazer, Eds.), Academic Press, New York, 1980, 149-159.

7. J. Mawhin, Contractive mappings and periodically perturbed conservative systems, Arch. Math. (Brno) 12 (1976), 67-73.

8. Semilinear equations of gradient type in Hilbert spaces and applications to differential equations, Rep. 139, Inst. Math. Pure Appl. Univ. Catholique de Louvain, October 1980.

9. M. M. Vainberg, Variational methods for the study of nonlinear operators, Holden-Day, San Francisco, Calif., 1964.

Department of Mathematics, Texas AkM University, College Station, Texas 77843 